\title{
Modification of Polyimide Wettability by KrF Pulsed Laser Ablation
}

\author{
V. Oliveira* and R. Vilar*
}

* Department of Materials Engineering, Instituto Superior Técnico, Av. Rovisco Pais nº 1, 1049-001 Lisboa, Portugal, vitor.oliveira@ist.utl.pt

Laser ablation has been extensively studied in polymers because of their considerable utility in diverse fields such as medicine, microelectronics, photonics, etc. As a result, laser ablation of polymers has generated considerable interest in research during the past two decades [1]. Among the many aspects of laser ablation of polymers, several authors have reported the formation and growth of conical structures during laser processing with UV laser radiation [2-5]. Although this aspect of laser ablation has been investigated since the 1980s, only a few applications of these structures have been reported. In the present paper, we study the development of conical structures on polyimide treated with $\mathrm{KrF}$ laser radiation as a function of the radiation fluence, and its effect on the wettability of the polymer.

Polyimide (Kapton $\mathrm{HN}^{\mathrm{TM}}$, Dupont) films (125 $\mu \mathrm{m}$ thick) were processed in air using a $\mathrm{KrF}$ excimer laser (Lambda Physik, Compex 110, $\lambda=248 \mathrm{~nm}, \tau=30 \mathrm{~ns}$ ). The laser beam was shaped by inserting an aperture mask into the beam path to select the central area, where it is fairly homogeneous. The mask was optically projected onto the sample surface by means a lens, to form a rectangle spot. The radiation fluence was evaluated from impact area and pulse energy measurements. Polyimide samples were irradiated with 500 pulses and radiation fluences ranging from 35 to $5000 \mathrm{~mJ} / \mathrm{cm}^{2}$, at a constant frequency of $10 \mathrm{~Hz}$. After irradiation, the surface morphology was investigated by scanning electron microscopy and the wettability measured by the contact angle method.

Representative SEM images illustrating the evolution with the radiation fluence of polyimide surfaces treated with 500 laser pulses are depicted in figure 1. For fluences above 50 and up to $\approx$ $500 \mathrm{~mJ} / \mathrm{cm}^{2}$, cone-like structures are formed. Densely packed cones are observed if the radiation fluence is slightly higher than the ablation threshold while sparsely distributed cones can be observed if high fluences are used. Also, the number of cones decreases with the radiation fluence and their base diameter increases. Figure 2 shows the variation of the average height of cones as a function of the radiation fluence. The height of the cones can vary from 10 to $60 \mu \mathrm{m}$, depending on the radiation fluence used. A slight decrease of the cones height is observed at $150 \mathrm{~mJ} / \mathrm{cm}^{2}$, an effect that can be explained by a change in the growth mechanism [6].

Photographs of water droplets on unprocessed polyimide and processed with 500 pulses at 200 $\mathrm{mJ} / \mathrm{cm}^{2}$ are shown in figure 3. It is evident that laser processing induces a remarkable change in the wettability of polyimide. The water contact angle of polyimide is about $70^{\circ}$. In contrast, after laser processing, this angle is about $140^{\circ}$. Changes in the observed wettability may be attributed to a synergy of surface chemistry and roughness effect.

In conclusion, modification of polyimide wettability can be achieved by $\mathrm{KrF}$ pulsed laser ablation and explained by an increase of the roughness of the irradiated surfaces. 


\section{References}

[1] P.E. Dyer, Appl Phys A, 77 (2003) 167.

[2] P.E. Dyer, S.D. Jenkins, J. Sidhu, Appl Phys Lett, 49 (1986) 453.

[3] D.J. Krajnovich, J.E. Vasquez, J Appl Phys, 73 (1993) 2001.

[4] F. Raimondi, S. Abolhassani, R. Brutsch, F. Geiger, T. Lippert, J. Wambach, J. Wei, A. Wokaun A, J Appl Phys, 88, (2000) 3659.

[5] N.S. Murphy, R.D. Prabhu, J.J. Martin, L. Zhou, R.L. Headrick, J Appl Phys, 100, (2006) 23538.

[6] V. Oliveira, R. Vilar, Appl Phys A, submitted.

[7] Wenzel, Ind Eng Chem, 28 (1936) 989.

[8] A.B. Cassie, S. Baxter, Trans Faraday Soc, 40 (1994) 546. 


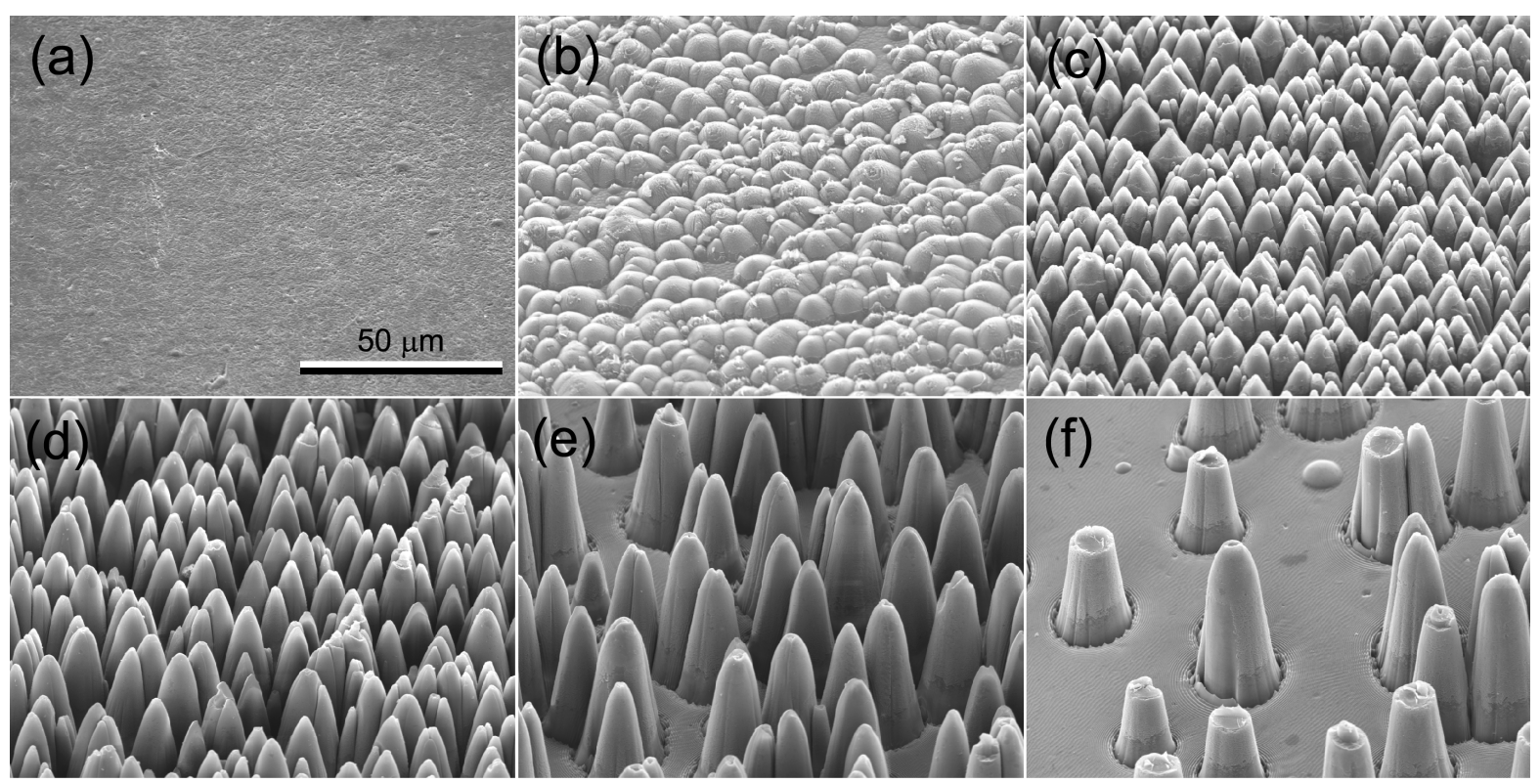

Figure 1. Surface morphology as a function of the radiation fluence. (a) 35; (b) 50; (c) 105; (d) 225; (e) 305 ; and (f) $420 \mathrm{~mJ} / \mathrm{cm}^{2}$. Number of pulses is 500 .

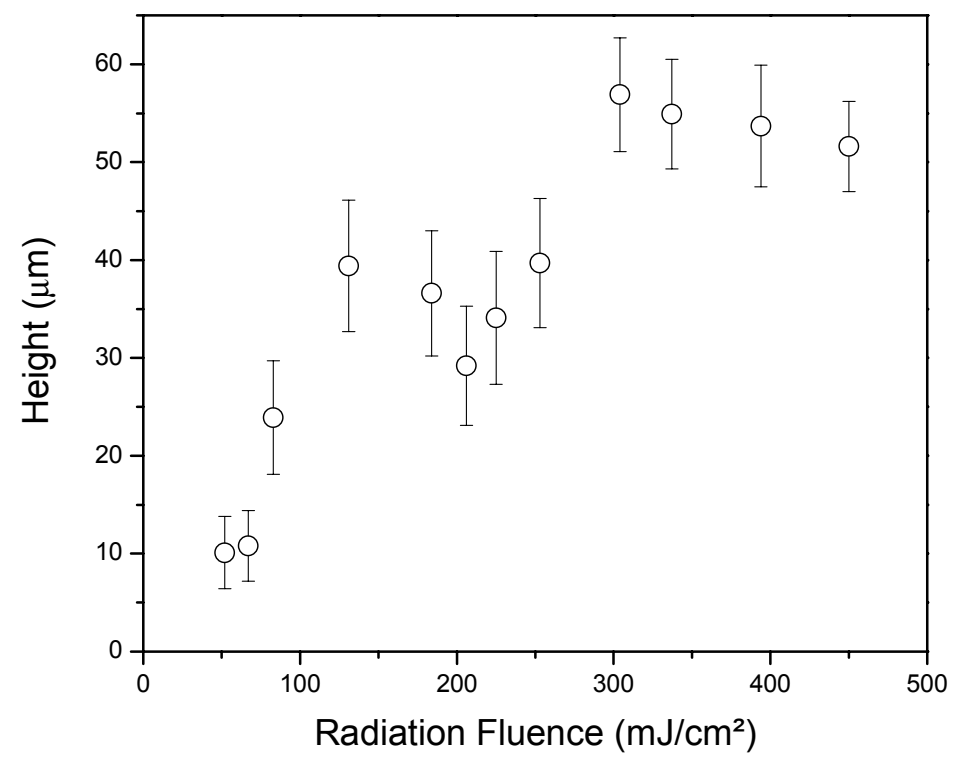

Figure 2. Average cones height as a function of radiation fluence. Number of pulses is 500 . 

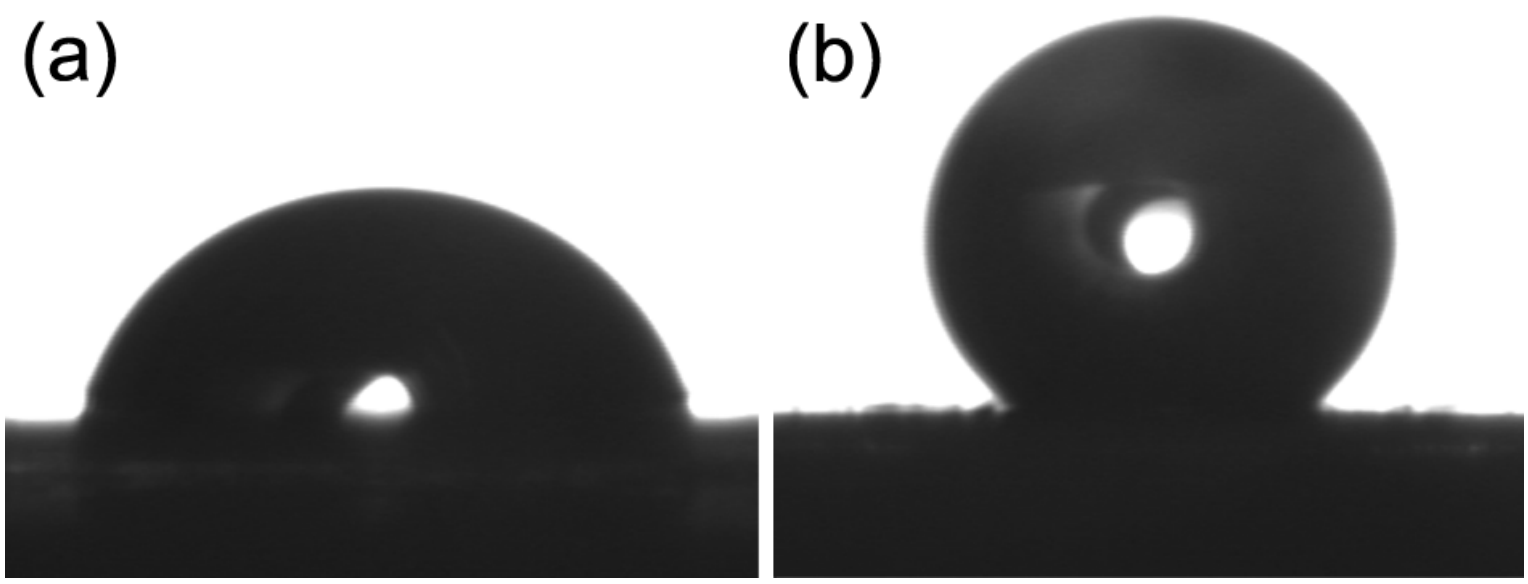

Figure 3. Photographs of water droplets on polyimide surfaces: (a) unprocessed; and (b) structured with 500 laser pulses a $200 \mathrm{~mJ} / \mathrm{cm}^{2}$. 